\title{
5th Grade Completion
}

National Cancer Institute

\section{Source}

National Cancer Institute. 5th Grade Completion. NCI Thesaurus. Code C67127.

Indicates that 5th grade is the highest level of educational achievement. 